\title{
Counting Lines and Conics on a Surface
}

\author{
Dedicated to Professor Friedrich Hirzebruch on his 80th birthday
}

By

\section{Yoichi MIYAOKA*}

\section{$\S 1 . \quad$ Main Results}

This short note is a supplementary remark to the author's article [7]. Our main results are the following two propositions concerning the number of rational curves and elliptic curves on polarized complex algebraic surfaces:

Proposition A. Let $X \subset \mathbb{P}_{\mathbb{C}}^{\frac{h}{2}+1}$ be a $K 3$ surface of degree $h \geq 4$ and let $r_{d}=r_{d}(X)$ denote the number of rational curves of degree $d$ on $X, d \in \mathbb{Z}_{>0}$. If $h>4 N^{2}$ for a positive integer $N$, then $r_{1}+2 r_{2}+\cdots+N r_{N} \leq \frac{24 N h}{h-4 N^{2}}$. In particular, $r_{1} \leq \frac{24 h}{h-4}$ for $h \geq 6$ and $r_{2} \leq \frac{24 h}{h-16}$ for $h \geq 18$.

Proposition B. Let $X \subset \mathbb{P}_{\mathbb{C}}^{N}$ be a canonically embedded surface of degree $K^{2}$ and put $\sigma=c_{2} / K^{2} \geq 1 / 3$ (as usual, $K$ and $c_{2}$ stand for the canonical divisor and the topological Euler number of $X)$. Let $r_{d}=r_{d}(X)$ be the number of rational curves of degree $d$ on $X$ and let $s=s(X) \in \mathbb{Z}_{\geq 0} \cup\{\infty\}$ denote the sum $\sum_{C} K C$ of the degrees of the elliptic curves $C \subset X$.

(1) Assume that $\sigma<1+\frac{4}{N}+\frac{6}{N^{2}}$ for some positive integer $N$. Then $\sum_{d=1}^{N} d r_{d} \leq \frac{(3 \sigma-1)\left(1+\frac{2}{N}\right)}{1-\sigma+\frac{4}{N}+\frac{6}{N^{2}}} K^{2}$. For instrance $r_{1} \leq \frac{9 \sigma-3}{11-\sigma} K^{2}$ if $\sigma<11$ (i.e., if $X$ is not a quintic $\subset \mathbb{P}^{3}$ ) and $r_{2} \leq \frac{6 \sigma-2}{9-2 \sigma} K^{2}$ if $\sigma<\frac{9}{2}$.

Communicated by S. Mori. Received April 11, 2008.

2000 Mathematics Subject Classification(s): Primary 14N15; Secondary 14J28, 14J29.

Partially supported by the JSPS Grant-in-Aid \# 19340003 "Minimal model theorem: its proof, development and applications"

* School of Mathematics, University of Tokyo, Komaba, Tokyo 153-8914, Japan.

(c) 2009 Research Institute for Mathematical Sciences, Kyoto University. All rights reserved. 
(2) If $\sigma<1$, then $s \leq \frac{3 \sigma-1}{1-\sigma} K^{2}$.

Proof of Propositions $A$ and $B$. Let $X$ be a smooth complex projective surface of non-negative Kodaira dimension. Pick a rational number $\alpha \in[0,1]$ and a finite sum $C=\sum C_{i}$ of distinct irreducible curves $C_{i} \subset X$ of geometric genera $g_{i}$. In [7], we defined an "orbibundle" $\widetilde{\mathcal{E}}_{\alpha}$ attached to the triplet $(X, C, \alpha)$ and showed the Miyaoka-Yau-Sakai inequality $3 c_{2}\left(\widetilde{\mathcal{E}}_{\alpha}\right) \geq c_{1}^{2}\left(\widetilde{\mathcal{E}}_{\alpha}\right)$ or, more explicitly,

(†) $\frac{\alpha^{2}}{2}\left(C^{2}+3 C K-6(g-1)\right)-2 \alpha(C K-3(g-1))+3 c_{2}-K^{2} \geq 0$.

Here $g-1$ is construed as the sum $\sum\left(g_{i}-1\right)$; see ibid., $\S 1$, Remark G.

Fix a very ample divisor $H$ of degree $h=H^{2}$ on $X$. Assume that $C_{i}$ is rational or elliptic (i.e., $g_{i}-1=-1$ or 0 ). The formula ( $\dagger$ ) involves four parameters $\alpha, C^{2}, C K$ and $g-1$ (= the number of rational curves with opposite sign). Denoting by $\delta$ the total degree $C H=\sum C_{i} H$, we bound $C^{2}$ by $\delta^{2} / h$ (the Hodge index theorem). This substitution simplifies ( $\dagger$ ) in two important cases:

Case A: $(X, H)$ is a polarized K3 surface $(K=0)$ of degree $h$. Then

$$
\alpha^{2}\left(\frac{\delta^{2}}{h}-6(g-1)\right)+12 \alpha(g-1)+144 \geq 0 \quad \text { for } \alpha \in[0,1] .
$$

CASE B: $X$ is a canonical surface $(H=K)$. For $\alpha \in[0,1]$, we have

$$
\alpha^{2}\left(\frac{\delta^{2}}{K^{2}}+3 \delta-6(g-1)\right)-2 \alpha(2 \delta-6(g-1))+6 c_{2}-2 K^{2} \geq 0 .
$$

We view the left hand sides of these two inequalities as quadratic functions $Q_{1}(\alpha), Q_{2}(\alpha)$ of $\alpha$, which attain the minima at $\alpha_{1}, \alpha_{2} \in[0,1] \cap \mathbb{Q}$. We readily elicit Propositions A and B from the inequalities $Q_{i}\left(\alpha_{i}\right) \geq 0, i=1,2$.

Remarks. (1) A general projective K3 surface $X$ is known to carry countably many nodal rational curves [3] as well as a one-parameter family of nodal elliptic curves [8]; i.e., $\sum_{d}^{\infty} d r_{d}(X)=\infty, s(X)=\infty$.

(2) The author has no idea how close to the best possible our estimates are. Nor does he know if there are any precedent results, apart from a handful of treatises that study either lines on surfaces in $\mathbb{P}_{\mathbb{C}}^{3}[11]$, [12], [2], [1] or configurations of disjoint smooth rational curves [6], [9], [10]. As shown in $\S \S 2$ and 3 , the inequality $(\dagger)$ is optimal for countably many examples. 
(3) Unfortunately, $(\dagger)$ does not say anything about the classical problem of counting lines on surfaces in $\mathbb{P}_{\mathbb{C}}^{3}$. We are luckier when dealing with lines on complete intersections of codimension two or more; elementary, but cumbersome, calculation of explicit bounds is left to the reader.

\section{§2. Two Examples}

Take four points $P_{1}, P_{2}, P_{3}, P_{4}$ in general position on $\mathbb{P}^{2}$. The line $L_{i j}$ connecting $P_{i}$ and $P_{j}$ is defined by a linear form $\lambda_{i j}$, and the ratios $\lambda_{i j} / \lambda_{k l}$ are rational functions on $\mathbb{P}^{2}$. Fixing a positive integer $n \geq 2$, the $n$-th roots of these ratios define a Kummer extension $K_{n}$ of $\mathbb{C}\left(\mathbb{P}^{2}\right)$ with Galois group $(\mathbb{Z} / n \mathbb{Z})^{\oplus 5}$ (cf. Hirzebruch [5]).

Let $X_{1} \rightarrow \mathbb{P}^{2}$ be the blowing up at the four points $P_{i} . X_{1}$ is a del Pezzo surface of degree five (unique up to isomorphisms). Denote by $E_{i} \subset X_{1}$ the exceptional curve over $P_{i}$ and let $\widetilde{L}_{i j} \subset X_{1}$ be the strict transform of the line $L_{i j} \subset \mathbb{P}^{2}$. The reduced curve $D=\bigcup_{i=1}^{4} E_{i} \cup \bigcup_{i, j} \widetilde{L}_{i j}$ is the union of all the $(-1)$-curves on $X_{1}$ and linearly equivalent to $-2 K_{X_{1}}$. D has 15 double points and its smooth part consists of 10 components, each of which isomorphic to $\mathbb{P}^{1}$ minus three points. The minimal model of the function field $K_{n}$ is realized as a finite covering $\pi_{n}: X_{n} \rightarrow X_{1}$ with branch locus $D$ of constant ramification index $n$. Standard invariants of $X_{n}$ are given by:

$$
K_{X_{n}}=\left(\frac{1}{2}-\frac{1}{n}\right) \pi_{n}^{*} D, \quad c_{2}\left(X_{n}\right)=n^{5}\left(2-\frac{10}{n}+\frac{15}{n^{2}}\right) .
$$

Specifically, $X_{5}$ is a ball quotient with $K^{2}=5^{4} \times 9=3 c_{2}$ (see $\left.i b i d\right)$.

The pullback of $E_{i}$ or $\widetilde{L}_{i j}$ via $\pi_{n}: X_{n} \rightarrow X_{1}$ is divisible by $n$ and supported by $n^{2}$ disjoint curves, each of which being isomorphic to the Fermat curve $x^{n}+y^{n}+z^{n}=0$ of genus $(n-1)(n-2) / 2$. The half of the ramification locus $H_{n}=R_{n} / 2=\pi_{n}^{*} D /(2 n)$ turns out to be an integral, very ample divisor on $X_{n}$. Thus i) $\left(X_{n}, H_{n}\right)$ is a polarized surface of degree $5 n^{3}$, ii) the ramification locus $R_{n}$ of $\pi_{n}: X_{n} \rightarrow X_{1}$ consists of $10 n^{2}$ irreducible components, all isomorphic to the Fermat curve of degree $n$, and iii) $K_{X_{n}}=(n-2) H_{n}$. By choosing 2 and 3 as values of $n$, we obtain two examples for which our upper bound of $r_{2}$ in Proposition A and that of $s$ in Proposition B are respectively attained:

Example A. $\left(\mathrm{X}_{2}, \mathrm{H}_{2}\right)$ is a $\mathrm{K} 3$ surface of degree 40 , with the effective divisor $R_{2} \sim 2 H_{2}$ consisting of 40 conics. Thus $r_{2}\left(X_{2}\right) \geq 40=\frac{24 \times 40}{40-16}$.

Example B. $\left(X_{3}, H_{3}\right)$ is a canonical surface with $K^{2}=3^{3} \times 5, c_{2}=$ $3^{3} \times 3$ (i.e., $\sigma=3 / 5$ ). The divisor $R_{3}$ is a union of 90 copies of the Fermat 
cubic curve, so that the total degree of the elliptic curves $s\left(X_{3}\right)$ is at least $90 \times 3=\frac{3 \sigma-1}{3(1-\sigma)} K^{2}$.

\section{$\S 3 . \quad$ A Concluding Remark}

The Hirzebruch proportionality principle explains why $(\dagger)$ turns into an equality for the examples in the previous section.

Let $\mathbb{B}^{2} \subset \mathbb{C}^{2}$ denote the unit ball $\mathrm{PU}(1,2) / \mathrm{P}(\mathrm{U}(1,1) \times \mathrm{U}(1))$ equipped with the Bergmann metric. Let $\Gamma_{0} \subset \mathrm{PU}(1,2)$ be a discrete, torsion-free, cocompact subgroup of the holomorphic isometries of $\mathbb{B}^{2}$. Consider a $\Gamma_{0}$-stable curve $\Delta \subset \mathbb{B}^{2}$ with only normal crossing singularities ( $\Delta$ may have countably many irreducible components). Let $\Gamma \subset \mathrm{PU}(1,2)$ be a subgroup which satisfies the following four conditions:

(1) $\Gamma$ contains $\Gamma_{0}$ as a normal subgroup with $\Gamma / \Gamma_{0} \simeq(\mathbb{Z} / m \mathbb{Z})^{\oplus r}$.

(2) $X_{1}=\Gamma \backslash \mathbb{B}^{2}$ is nonsingular.

(3) The action of $\Gamma$ preserves $\Delta$.

(4) The projection $\pi: Y=\Gamma_{0} \backslash \mathbb{B}^{2} \rightarrow X_{1}=\Gamma \backslash \mathbb{B}^{2}$ is a Kummer cover branching along $D=\Gamma \backslash \Delta \subset X_{1}$ with constant ramification index $m$.

Then $D \subset X_{1}$ is necessarily a divisor with only normal crossings. The "orbibundle" $\widetilde{\mathcal{E}}_{1-\frac{1}{m}}$ on $X$ constructed in [7] from the pair $\left(X_{1}, D\right)$ is identified with $\Omega_{Y}^{1}$ in this case, so that $c_{1}^{2}\left(\widetilde{\mathcal{E}}_{1-\frac{1}{m}}\right)=3 c_{2}\left(\widetilde{\mathcal{E}}_{1-\frac{1}{m}}\right)$ by the Hirzebruch proportionality theorem [4]. If there is another Kummer cover $p_{n}: X_{n} \rightarrow X_{1}$ branching along the same divisor $D$, but with a smaller ramification index $n<m$, then the same orbibundle $\widetilde{\mathcal{E}}_{1-\frac{1}{m}}$ on $\left(X_{1}, D\right)$ can be viewed as the orbibundle $\widetilde{\mathcal{E}}_{1-\frac{n}{m}}$ associated with $\left(X_{n}, R_{n}\right)$, where $R_{n}=p_{n}^{*} D / n$ is the ramification locus of $X_{n} \rightarrow X_{1}$. This is precisely the case in Examples A and B, where $(m, n)=(5,2)$ and $(5,3)$. Recalling that the inequality $(\dagger)$ is essentially the Miyaoka-Yau-Sakai inequality $3 c_{2}\left(\widetilde{\mathcal{E}}_{\alpha}\right) \geq c_{1}^{2}\left(\widetilde{\mathcal{E}}_{\alpha}\right)$, we see that $(\dagger)$ is indeed an equality when $(X, C, \alpha)=\left(X_{n}, R_{n}, \frac{m-n}{m}\right)$.

The construction above produces countably many examples of $(X, C, \alpha)$ to which the attached orbibundle $\widetilde{\mathcal{E}}_{\alpha}$ satisfies $3 c_{2}\left(\widetilde{\mathcal{E}}_{\alpha}\right)=c_{1}^{2}\left(\widetilde{\mathcal{E}}_{\alpha}\right)$. It is another question, however, if we can find infinitely many such triples with $C$ being a union of curves of small genera (a union of rational or elliptic curves, for example). 


\section{References}

[1] S. Boissière and A. Sarti, Counting lines on surfaces, Ann. Sc. Norm. Super. Pisa Cl. Sci. (5) 6 (2007), no. 1, 39-52.

[2] L. Caporaso, J. Harris and B. Mazur, How many rational points can a curve have?, in The moduli space of curves (Texel Island, 1994), 13-31, Progr. Math., 129, Birkhäuser, Boston, Boston, MA, 1995.

[3] X. Chen, Rational curves on K3 surfaces, J. Algebraic Geom. 8 (1999), no. 2, 245-278.

[4] F. Hirzebruch, Automorphe Formen und der Satz von Riemann-Roch, in Symposium internacional de topología algebraica International symposium on algebraic topology, 129-144, Universidad Nacional Autónoma de México and UNESCO, Mexico City, 1958.

[5] - Arrangements of lines and algebraic surfaces, in Arithmetic and geometry, Vol. II, 113-140, Progr. Math., 36, Birkhäuser, Boston, Mass, 1983.

[6] Y. Miyaoka, The maximal number of quotient singularities on surfaces with given numerical invariants, Math. Ann. 268 (1984), no. 2, 159-171.

7] $\_$, The orbibundle Miyaoka-Yau-Sakai inequality and an effective BogomolovMcQuillan theorem, Publ. Res. Inst. Math. Sci. 44 (2008), no. 2, 403-417.

[8] S. Mori and S. Mukai, The uniruledness of the moduli space of curves of genus 11, in Algebraic geometry (Tokyo/Kyoto, 1982), 334-353, Lecture Notes in Math., 1016, Springer, Berlin, 1983.

[9] S. Rams, Three-divisible families of skew lines on a smooth projective quintic, Trans. Amer. Math. Soc. 354 (2002), no. 6, 2359-2367 (electronic).

[10] , Projective surfaces with many skew lines, Proc. Amer. Math. Soc. 133 (2005), no. 1, 11-13 (electronic).

[11] B. Segre, The maximum number of lines lying on a quartic surface, Quart. J. Math., Oxford Ser. 14 (1943), 86-96.

[12] On arithmetical properties of quartic surfaces, Proc. London Math. Soc. (2) 49 (1947), 353-395. 